\title{
Concept of Bee-Eyes Array of Fresnel Lenses as a Solar Photovoltaic Concentrator System
}

\author{
Nura Liman Chiromawa ${ }^{1,2}$ and Kamarulazizi Ibrahim ${ }^{1}$ \\ ${ }^{1}$ Nano-Optoelectronics Research and Technology Laboratory, School of Physics, Universiti Sains Malaysia (USM), \\ 11800 Penang, Malaysia \\ ${ }^{2}$ Department of Physics, Umaru Musa Yar'adua University, PMB 2218, Katsina, Nigeria
}

Correspondence should be addressed to Nura Liman Chiromawa; nchiromawa@gmail.com

Received 3 October 2014; Revised 10 February 2015; Accepted 15 February 2015

Academic Editor: Patrick Kung

Copyright (c) 2015 N. L. Chiromawa and K. Ibrahim. This is an open access article distributed under the Creative Commons Attribution License, which permits unrestricted use, distribution, and reproduction in any medium, provided the original work is properly cited.

\begin{abstract}
This paper presents a proposal of a new configuration of an optical concentrator for photovoltaic application which may enhance the efficiency of solar cells. Bee-eyes array Fresnel lenses concentrator proposed here provide high concentration factor which is greater than $1000 \mathrm{x}$ at the 20th zone. In addition, the system also provides room for increasing the number of zones to achieve the high concentration factor if needs arise. The transmission efficiency greater than $90 \%$ has been achieved with $f$-number of $\geq 1.25$. Mathematical relations derived to obtain flux distribution at the absorber plane and the transmission efficiency as well as the position of the solar cell were used in the ray tracing simulations for $6,18,36,60,90,126,168,216,270$, and 330 suns concentration systems. A transmission efficiency is linearly decreasing with the increase in the number of arrays in which the transmission efficiency of $94.42 \%$ was recorded at the array of 6 suns and $74.98 \%$ at 330 suns.
\end{abstract}

\section{Introduction}

Solar photovoltaic (SPV) technology is one of the most attractive renewable energy options for energy generation due to its advantages over other energy options and the fact that it is very friendly to the environmental conditions, which requires only capital cost and less maintenance cost. However, it is limited because of its high cost per kilowatts-hour [1].

Crystalline silicon is the main component used in the fabrication of solar cells in SPV technology whereby the Sun's light energy is converted into electrical energy. Researches all over the world are continuing in looking for measures to produce highly efficient, low-cost solar cells [2]. Among such means are the use of thinner wafer [3], thin film solar cell technology [4], and optical concentration technologies [5]. Optical concentrator photovoltaic systems reduce the surface area of the solar cells required and increase the radiation intensity on the solar cells; thus, the solar cells with smaller surface area can generate high output power at lower cost of the entire system when compared with solar cells without a concentrator [6]. An optical concentrator photovoltaic system employs mirrors or lenses and/or a compound arrangement of both (mirrors and lenses) in a specified method of arrangement.

Generally, reflector-based concentrator systems like a parabolic dish/trough or a compound parabolic reflector reflect nonuniform solar radiation on the receiver/collector and they occupy larger space in comparison with the solar cells' surface area [6, 7]. Hence, the refractor-based concentrators which usually use Fresnel lenses may be found to be more suitable because of some advantages which they have over reflectors. A Fresnel lens used in concentrator photovoltaic applications is almost universally plano-convex in which solar radiations are focused by means of a series of concentric grooves (also referred to as point focus) or parallel grooves (known as line focus). When parallel rays of light are passing through the aperture of the Fresnel lens, each ring of the prisms refracts the light at a slightly different angle and focuses on a focal point.

Fresnel lenses find very wider applications in modern actively developing photovoltaic systems and they are becoming the backbone of the solar concentrators in different 
photovoltaic solar cells and panels. Developments of Fresnel lens for photovoltaic concentrators have been studied. In recent years, several methods of photovoltaic concentrators with Fresnel lenses using different materials have been investigated.

Chen and Su have presented a simulation result on Fresnel lens and secondary optical elements as a concentrator PV system by the ray tracing method using ASAP software, in which the comparison between the estimated optical efficiencies and the acceptance angles of a Fresnel lens concentrator (i.e., without secondary optical element SOE), a Fresnel lens concentrator with reflective pyramid as a SOE, a Fresnel lens concentrator with a reflective cone type as a SOE, and a Fresnel lens concentrator with a refractive dome type as a SOE are drawn, respectively $[7,8]$.

Miñano et al. designed and fabricated a multifunctional concentrator PV system called Fresnel Köhler (FKconcentrator) by Köhler integration principles, in which a PMMA Fresnel lens with four identical quadrants (as a primary optical element POE), along with a free-form glassmolded lens with four identical sectors (as a secondary optical element SOE), is arranged in the form of Köhler array. Thus, each pair of a quadrant and a sector works together as a Köhler integrator couple [9].

On the other hand, Languy et al. demonstrate the feasibility of obtaining a Fresnel lens with high concentration ratio operating with wavelengths between $380 \mathrm{~nm}$ and $1600 \mathrm{~nm}$ via polymethylmethacrylate (PMMA) and polycarbonate PC in which the linear chromatic aberration of singlet and doublets hybrid lenses was compared [10].

Another research led by Kusko et al. entails simulating a Fresnel lens and binary phase gratings via glass of refractive index $(n=1.5)$ by the beam propagation method. They compared their result with the theoretical values and found that it was close to theoretical values [11].

In a related development, Jing et al. study the optical performance of the compound Fresnel lens as a concentrator photovoltaic system comprising sawteeth Fresnel lens as a primary optical element and ring type Fresnel lens as a secondary optical element. In line with this, the improvements in the acceptance angle, the uniformity in the solar radiation on the solar cell, and the reducing of the aspect ratio were significantly studied. Thus, higher optical efficiency and smaller aspect ratio were achieved [12].

Previous researches on the concentrator photovoltaic systems suffer from nonuniformity in the solar flux distribution on the solar modules/panels causing the system's efficiency to be low. The concept of mimicking nature in the development of physical science has already been introduced [13]. Hence, the concept of mimicking nature in the development solar photovoltaic concentrator should not be ignored. In this paper, a new model of a multilevel Fresnel lens concentrator photovoltaic system that mimics natural Bee-eyes structural arrangement is introduced. The solar flux distribution and the optical concentration efficiency on the module are investigated using simple ray-tracing simulations and the principles of the superposition theory. The result obtained shows that this model can provide a uniform flux distribution of the solar radiation throughout the surface of the target area (solar cells) with high concentration ratio. Thus, it can be used to improve the efficiency of the solar photovoltaic generator.

\section{Conceptual Design of Fresnel Lenses in Bee-Eyes Array for PV Concentrator}

Because of their aspheric shape and thinness, leading them to be free from spherical aberration with very low absorption loss, large aperture Fresnel lenses are used in solar photovoltaic concentrator systems. Conventional Fresnel lens concentrator photovoltaic systems were generally designed to focus a collimated beam of solar irradiance to a given point or an area within the surface of solar module/panel. Thus, the irregular illumination on the surface of solar module/panel causes the reduction of the maximum power extraction. Nonuniformity in the illumination creates a significant localized hot spot on the solar module/panel, thus lowering the efficiency and reducing the lifetime of the solar cells $[7,12,14]$.

The problems of producing low conversion efficiency due to irregular illumination of solar irradiance may be addressed by using solar cells that are uniquely designed for a particular optical concentration photovoltaic system. This also has a disadvantage in that the design of the cells/module/panel has to be done for a particular optical concentration photovoltaic system. This could be addressed by way of introducing a new technique that mimics nature using Fresnel lenses arranged in the Bee-eyes array format.

Figure 1(a) shows the two-dimensional view of Beeeyes array of Fresnel lenses concentrator system which is described above as an example. The central lens block does not require any facet; this is because the light is not expected to be bent in this region. The system uses hexagonal lens blocks in which series of blocks at each zone are fixed at a very small angle of inclination and serves as sources of beam focusing onto the solar cell. This is to allow high concentration of solar irradiance at the target area (solar cell), while Figure 1(b) shows the 3-dimensional concentrator $\mathrm{PV}$ system in which the working principle is based on superposition theory. At any given zone, an array of Fresnel lenses along each side of hexagon is inclined at a small angle and bends the incident beam of the solar radiation onto the surface of the solar cells in such a way that the dimension of each hexagonal lens block is the same or is slightly greater than that of the solar cell. The superposition theory can properly be implemented if the inclination for each illumination lens block is in the direction normal to the vector, starting from its center, and this can be achieved by the use of the inclination angle of each illumination zone.

The Bee-eyes multilevel Fresnel lens concentrator system has a regular hexagonal shape with an array of regular hexagonal Fresnel lenses serially along each side of the hexagon. If $n$ is the number of a given zone corresponding to a level starting from the optical center of the system, then for the first zone corresponding to level one, the system will have an array of 6 -Fresnel lenses. Therefore, there is $(6+6(n-1))$ array of Fresnel lenses expanding hexagonally from the optical center of this system. 


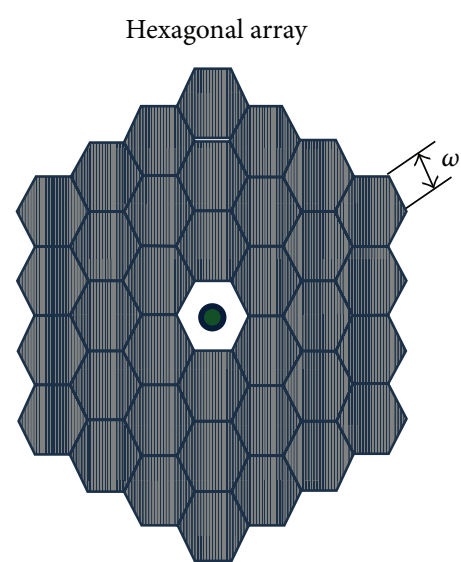

(a)

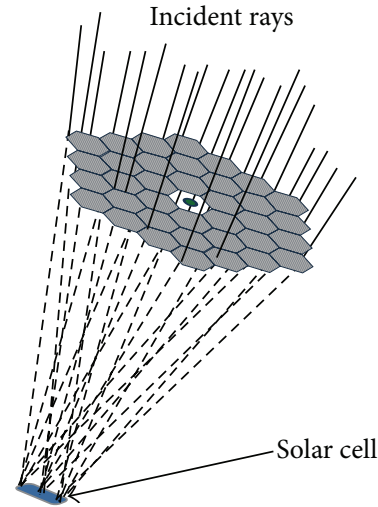

(b)

Figure 1: Concept of Bee-eye array of Fresnel lenses for solar concentrator PV system: (a) two-dimensional view of Bee-eye array of Fresnel lenses concentrator system and (b) three-dimensional view of concentrator PV system.

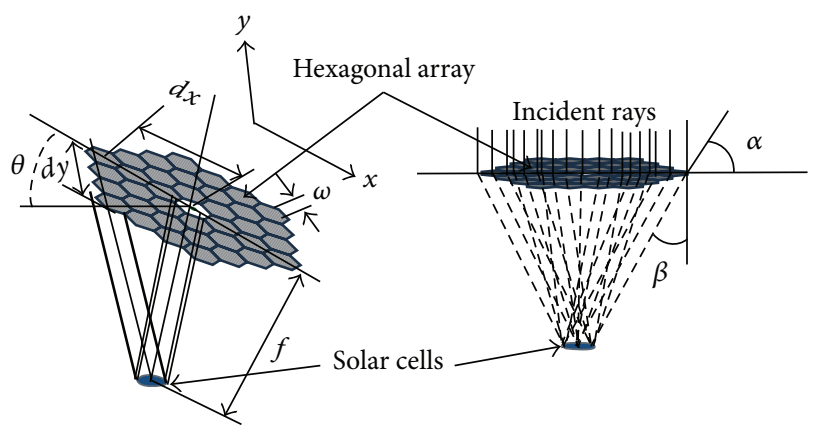

(a)

(b)

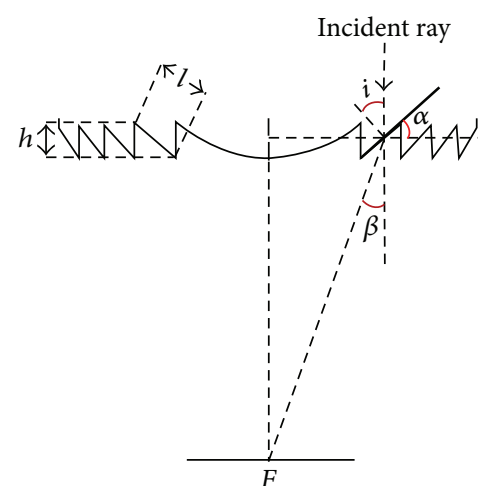

(c)

FIGURE 2: (a) Optical parameters of the concentrator PV system (b) and (c) cross sectional view of the Bee-eyes array of Fresnel lenses for the concentrator PV system.

From Figure 2(a), if the length of one side of a hexagonal block is denoted by $\omega$, then the total length of the system becomes

$$
\sum_{n=1}^{N}\{6(6 \omega+6 \omega(n-1))\}
$$

Let $i, j$ represent the $i$ th and $j$ th elements extending from $-n$ to $n$ in $x$-and $y$-axes; then the distance from the optical center of the array to the center of a hexagonal block in $x$ and $y$ will be given as $\omega \sqrt{3} i$ or $\omega \sqrt{3} j$.

The radiation beam incident normal at the surface of the lenses is refracted at an angle $\beta$ at the facets underneath the plane of the lens (as depicted in Figure 2(b)). Therefore, for any given hexagonal block with a central point distance of $\omega \sqrt{3} i$ or $\omega \sqrt{3} j$ from the optical centre of the array, the angle of refraction, $\beta(i, j)$, can be expressed as

$$
\begin{aligned}
\beta(i, j) & =\tan ^{-1}\left(\frac{\sqrt{\left\{(\omega i \sqrt{3})^{2}+(\omega j \sqrt{3})^{2}\right\}}}{f}\right) \\
& =\tan ^{-1}\left(\frac{\omega \sqrt{3} \times \sqrt{\left(i^{2}+j^{2}\right)}}{f}\right),
\end{aligned}
$$

where $f$ is the focal length of the concentrator system; however, in Figure 2(c) the sloped facets angle, $\alpha(i, j)$, can be obtained from Snell's law of refraction as

$$
\alpha(i, j)=\sin ^{-1}\left(\sqrt{\frac{\sin ^{2} \beta(i, j)}{\left(n^{2}-2 n \cos \beta(i, j)+1\right)}}\right),
$$

where $n$ is the refractive index of the material used for the fabrication of the Fresnel lens.

\section{Determination of the Performance of the System}

The transmission efficiency, the optical concentration ratio, and the uniformity of solar fluxes distribution on the surface of the solar cells were determined using the ray tracing simulation. Polymethylmethacrylate (PMMA) was selected 


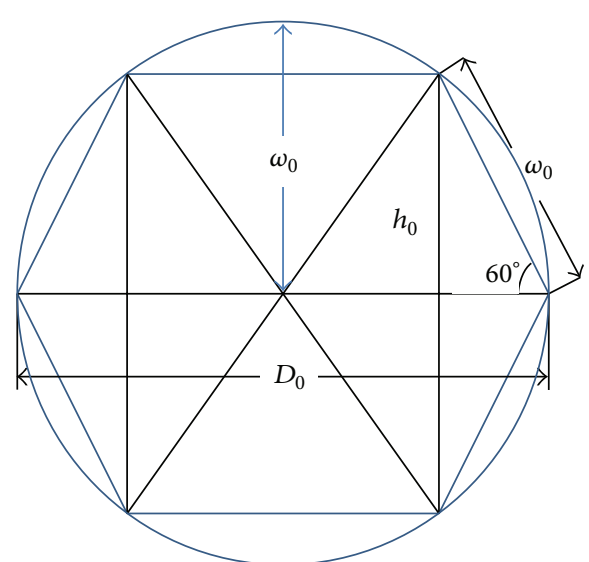

(a)

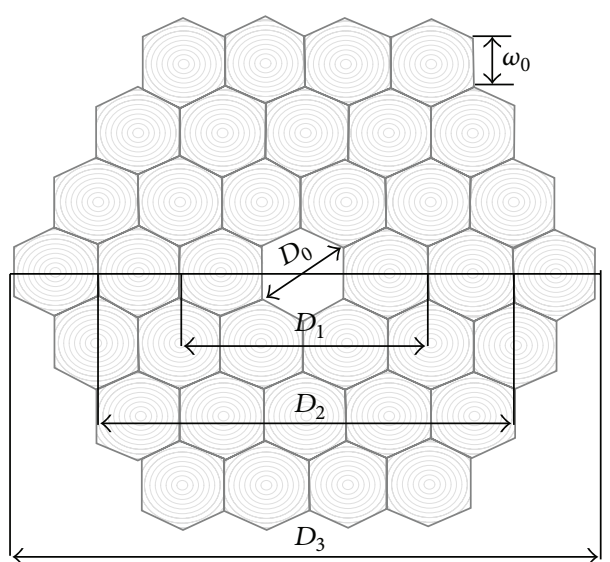

(b)

FIGURE 3: Geometric structures defining the geometrical parameters of the lens.

as lens materials, as PMMA has some good optical properties in the solar spectrum of the range of the wavelength between $0.23 \mu \mathrm{m}$ and $0.314 \mu \mathrm{m}$. In addition to this, PMMA is a lightweight material with shatter resistance, low cost, and available material, with high transmission efficiency in the wavelength ranging between $0.38 \mu \mathrm{m}$ and $0.78 \mu \mathrm{m}$. The refractive index of PMMA is 1.4914 at $0.5876 \mu \mathrm{m}$, and, therefore, this refractive index was used in the determination of angles of the sloped facet and the respective transmission efficiencies. Similarly, to include the contributions of the photons to the conversion of energy, the simulation was done by multiplying the sum of flux distribution of the solar spectrum and the product of irradiance on the earth surface and the spectral response of crystalline silicon solar cells $[2,15]$.

The angular diameter of the sun is 30 arcmin, and the angular extension of the sun with respect to the position of the concentrator PV system plays a vital role in limiting the uniformity of flux distribution at the absorber. This problem was addressed by applying the convolution to the flux distribution with a kernel function, in which the kernel function was given as two-dimensional arrays with constant value within a circle whose diameter corresponds to $f \phi_{\text {sun }}$.

The ray tracing simulation was done by varying the angle of incidence, $f$-number, and optical concentration ratio. The length of one side of the hexagonal array $\omega$ was fixed at $\omega=1 \mathrm{~m}$, and the focal lens of the system varied between $0.75 \mathrm{~m}$ and $3 \mathrm{~m}$, while the angle of incidence ranges between $0^{\circ}$ and $5^{\circ}$, and the solar cells with hexagonal shape having the same area as one of the hexagonal blocks of the array was adopted for simulation. The length of the sloped facet $l$ was approximated to $\omega / 52$ and the height of the facet $h$ at any given point in $(i, j)$ can be obtained by the following equation:

$$
h(i, j)=l(i, j) \tan \alpha(i, j)
$$

The transmission of light at the immediate surface of Fresnel lens $T_{\text {IS }}$ can be obtained by assuming that the angle of incidence to be very negligible $\left(\emptyset_{i} \approx 0\right)$ from the Fresnel equation as expressed below:

$$
T_{\text {IS }}=\frac{4 n}{(n+1)^{2}}
$$

On the other hand, for a given Fresnel lens with a slope facet angle $\alpha$ and a transmission angle $\beta$, the surface transmission $T_{\mathrm{RS}}$, at the rear surface, can be expressed as [14]

$$
\begin{aligned}
& T_{\mathrm{RS}} \\
& =\left(\sqrt{\frac{1}{2}\left(1-\frac{\tan ^{2} \beta}{\tan ^{2}(2 \alpha+\beta)}\right)^{2}+\left(1-\frac{\sin ^{2} \beta}{\sin ^{2}(2 \alpha+\beta)}\right)^{2}}\right) .
\end{aligned}
$$

For a given length of a side of lens block as $\omega$, the diagonal as $D$, and the perpendicular height $h$, as depicted in Figures 3 (a) and 3(b), the geometrical concentration ratio GCR of the array system can be estimated using

$$
\mathrm{GCR}=\frac{\left(\omega_{n}+D_{n}\right) h_{n}}{\left(\omega_{o}+D_{o}\right) h_{o}},
$$

where the subscript $o$ represents the dimensional parameters of solar cells and $n$ is the number zone in the array of Fresnel lenses' concentrator system. Thus, the optical concentration ratio OCR can be expressed in

$$
\mathrm{OCG}=\mathrm{GCR} \times T_{\mathrm{RS}} .
$$

The losses that resulted from the reflections of light at the surfaces of the lens are included by weighing the traced ray bundles by (5) and (6) and the uniformity of distributed flux at the absorber plane is also investigated by comparing the relative deviation (which is the ratio of the standard deviation of the solar irradiance to the maximum solar irradiance), within the surface area of solar cell. The flux distributions (for each wavelength $\lambda$ ) from the hexagonal lens blocks 


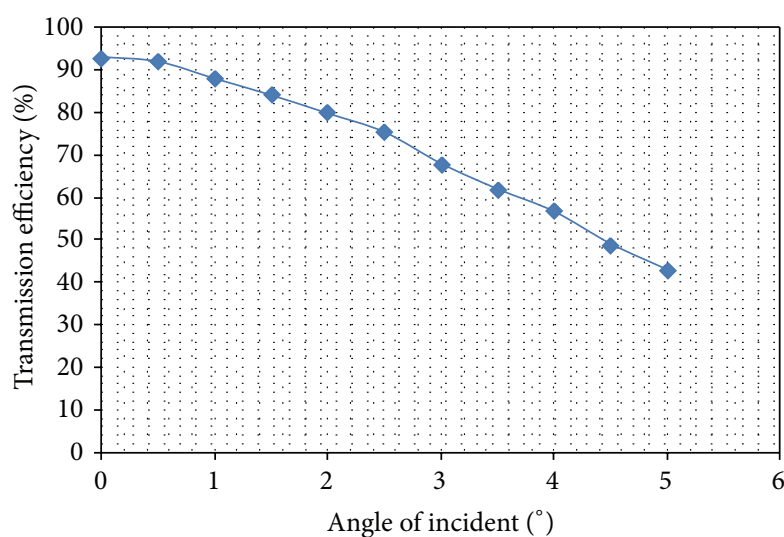

FIGURE 4: Transmission efficiency $T(\%)$, against incidence angle $\emptyset_{i}$ $\left({ }^{\circ}\right)$.

are estimated independently and are summed to estimate the performance at the position of the solar cell. The flux distribution at the absorber plane (i.e., at the surface of the solar cells) is the sum of the incidence fluxes of the individual lens blocks of the entire array multiplied by transmission efficiency $T_{\mathrm{RS}}$. Therefore, if $\phi_{\lambda}$ is denoted by monochromatic flux distribution at the surface of the solar cell formed by lens block, then the total flux distribution can be expressed as

$$
\begin{aligned}
\phi_{(\lambda)} & =T_{\mathrm{RS}} \sum_{\lambda} \varphi_{(\lambda)}(6+6(n-1)) \\
& =T_{\mathrm{RS}} \sum_{\lambda} \varphi_{(\lambda)} \sum_{n=1}^{N}(6+6(n-1)) .
\end{aligned}
$$

The effective transmission versus the angle of incidence is given in the graph of Figure 4. As seen, the transmission is decreasing with the increase in the incidence angle, where this shows that high transmission can be obtained at small angle of incidence. Meanwhile, the optical concentration ratio increases with the increase in the geometric concentration ratio which is exponentially increasing by the increase in number of zones, as depicted in Figure 5. On the other hand, the transmission efficiency is slightly decreasing with the increase in the number of arrays of Fresnel lenses (as seen in Figure 6). This due to the fact that as the number of array increased, the number of lines joining the hexagonal block also increased, thereby absorbing some of the incidence solar irradiance. In addition, the increase in the number of Fresnel zones and their corresponding levels causes the occurrence of the nonuniformity in the flux distribution at the absorber plane. Other factors include thermal expansion resulting from the rapid increase in the incident solar radiation as well as stray light on the aperture of the lens system.

The highest transmission efficiency corresponding to the lowest concentration ratio was achieved at zone 1 which is 6 suns. Conversely, the lowest transmission efficiency corresponding to the highest concentration ratio was achieved at zone 20 which is 330 suns.

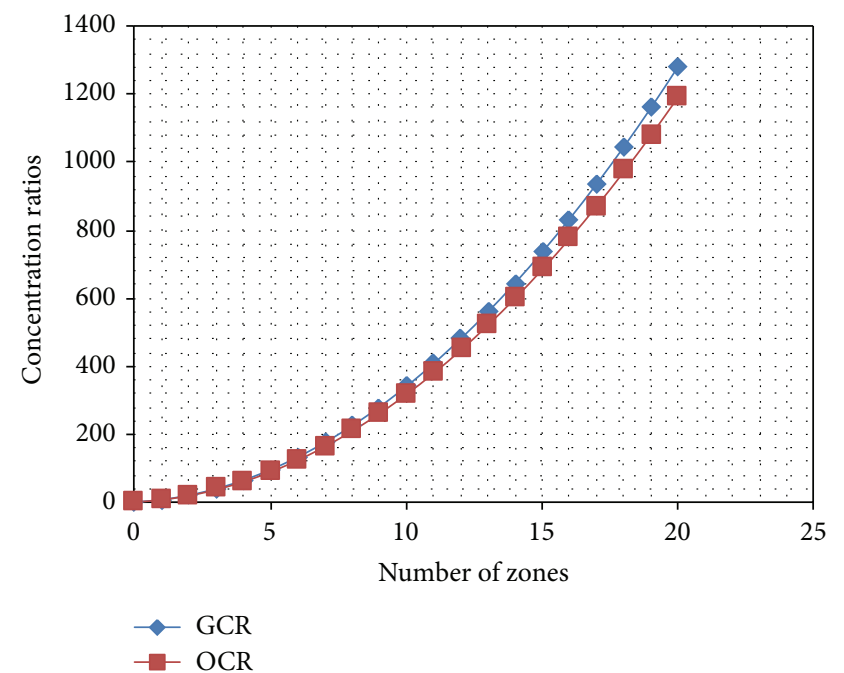

FIGURE 5: Optical and geometric concentration ratios against number of zones.

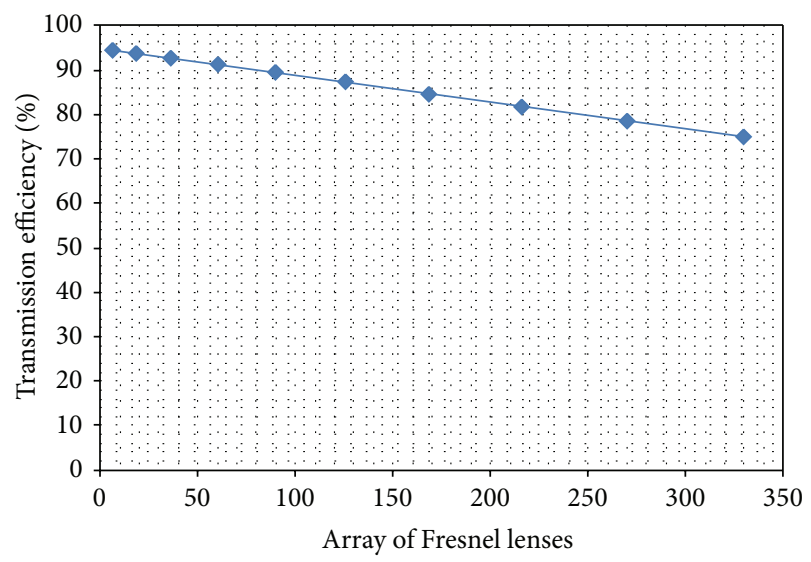

FIGURE 6: Transmission efficiency against array of Fresnel lenses.

\section{Conclusion}

The new concept of Bee-eyes array of Fresnel lenses for solar photovoltaic concentrator system introduced in this paper is expected to improve the efficiency of solar cells at a reasonable cost with high concentration ratio at uniform illumination. Mathematical treatments used to evaluate the effective performance of the system with illustrations are presented.

The transmission efficiency and optical concentration ratio of the system with the number of zones ranging from 1 to 20 were evaluated. The transmission efficiency greater than $75 \%$ can be maintained with acceptance angle larger than $1.5^{\circ}$ where this shows that hexagonal Fresnel lenses are suitable for moderate concentrated photovoltaic systems.

With this system also, concentration factor greater than $1000 \mathrm{x}$ can be obtained with the number of zones greater than 15. However, the concentration increases with the increase in the number of zones; thus, the Bee-eyes array Fresnel lenses concentrator can be regarded as a nonlimiting concentration ratio and, therefore, it can be used to achieve moderate concentration at an affordable cost. 


\section{Conflict of Interests}

The authors declare that there is no conflict of interests regarding the publication of this paper.

\section{Acknowledgments}

The authors wish to acknowledge the financial support from the Universiti Sains Malaysia and Malaysian Ministry of Higher Education (MOHE) via their Fundamental Research Grant Scheme (FRGS) with Grant (203/PSF/6721001).

\section{References}

[1] C. S. Sangani and C. S. Solanki, "Experimental evaluation of Vtrough (2 suns) PV concentrator system using commercial PV modules," Solar Energy Materials and Solar Cells, vol. 91, no. 6, pp. 453-459, 2007.

[2] H. Ibrahim and I. Kamarulazizi, "Low cost fabrication for high efficiency monocrystalline silicon solar cells," in Proceedings of the 3rd World Conference on Photovoltaic Energy Conversion, pp. 1519-1520, Osaka, Japan, May 2003.

[3] R. M. Chyad, M. Z. Mat Jafri, and K. Ibrahim, "Fabricated nanofiber diameter as liquid concentration sensors," Results in Physics, vol. 3, pp. 91-96, 2013.

[4] B. Sopori, "Thin-film silicon solar cells," in Handbook of Photovoltaic Science and Engineering, A. Luque and S. Hegedus, Eds., pp. 307-357, John Wiley \& Sons, 2003.

[5] K.-K. Chong, S.-L. Lau, T.-K. Yew, and P. C.-L. Tan, "Design and development in optics of concentrator photovoltaic system," Renewable \& Sustainable Energy Reviews, vol. 19, pp. 598-612, 2013.

[6] Y. Wu, P. Eames, T. Mallick, and M. Sabry, "Experimental characterisation of a Fresnel lens photovoltaic concentrating system," Solar Energy, vol. 86, no. 1, pp. 430-440, 2012.

[7] Y. C. Chen and C. H. Su, "Concentrator design of a Fresnel lens and a secondary optical element," in Proceedings of the 6th International Conference on Concentrating Photovoltaic Systems (CPV'10), 2010.

[8] M. Z. Shvarts and A. A. Soluyanov, "Improved concentration capabilities of flat-plate Fresnel lenses," Advances in Science and Technology, vol. 74, pp. 188-195, 2011.

[9] J. C. Miñano, P. Benítez, P. Zamora, M. Buljan, R. Mohedano, and A. Santamaría, "Free-form optics for Fresnel-lens-based photovoltaic concentrators," Optics Express, vol. 21, no. 3, pp. A494-A502, 2013.

[10] F. Languy, K. Fleury, C. Lenaerts et al., "Flat Fresnel doublets made of PMMA and PC: combining low cost production and very high concentration ratio for CPV," Optics Express, vol. 19, supplement 3, pp. A280-A294, 2011.

[11] M. Kusko, D. Cristea, and P. Schiopu, "Simulation of fresnel lenses and binary phase gratings with beam propagation method," UPB Scientific Bulletin, Series C: Electrical Engineering, vol. 71, no. 2, pp. 55-62, 2009.

[12] L. Jing, H. Liu, H. Zhao et al., "Design of novel compound fresnel lens for high-performance photovoltaic concentrator," International Journal of Photoenergy, vol. 2012, Article ID 630692, 7 pages, 2012.

[13] B. Tian, X. Zheng, T. J. Kempa et al., "Coaxial silicon nanowires as solar cells and nano-electronic power sources," Nature, vol. 449, no. 7164, pp. 885-889, 2007.
[14] K. Ryu, J.-G. Rhee, K.-M. Park, and J. Kim, "Concept and design of modular Fresnel lenses for concentration solar PV system," Solar Energy, vol. 80, no. 12, pp. 1580-1587, 2006.

[15] C.-C. Tsui, H.-C. Wei, W.-F. Chang, and G.-D. J. Su, “Design and fabrication of a mid-wavelength infrared Fresnel lens via liquid poly(methyl methacrylate)," Journal of Micromechanics and Microengineering, vol. 22, no. 4, Article ID 045010, 2012. 

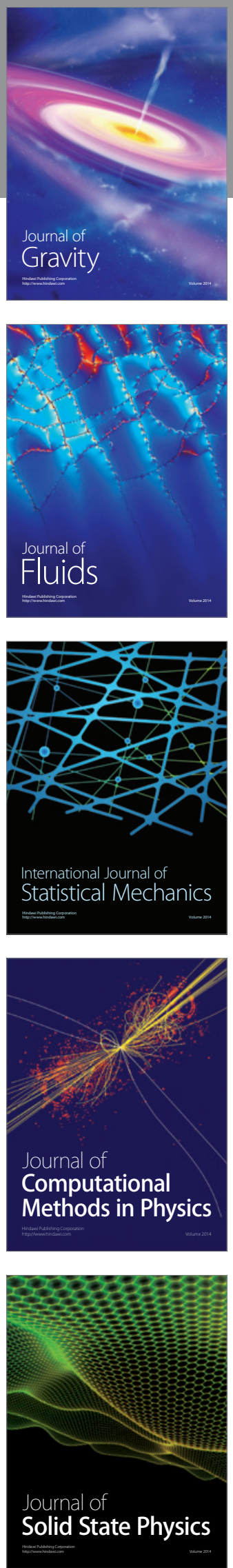

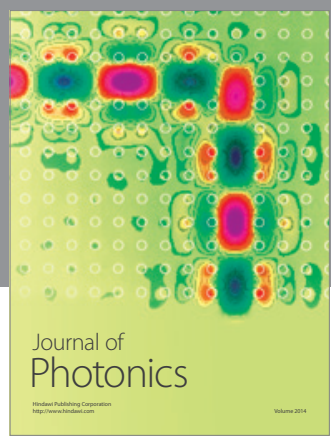

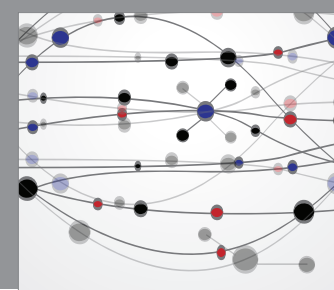

The Scientific World Journal

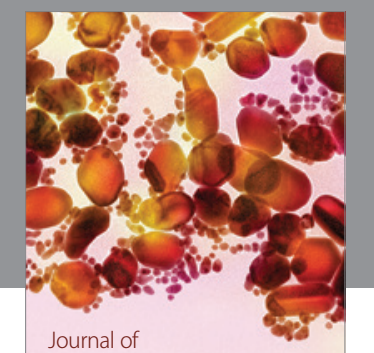

Soft Matter
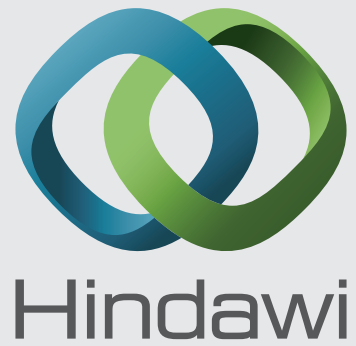

Submit your manuscripts at

http://www.hindawi.com
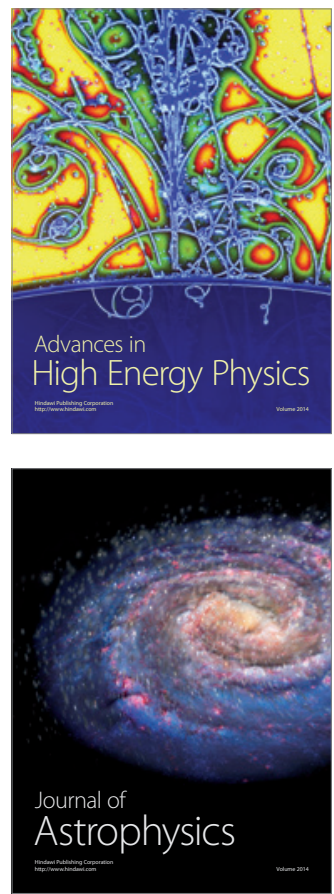
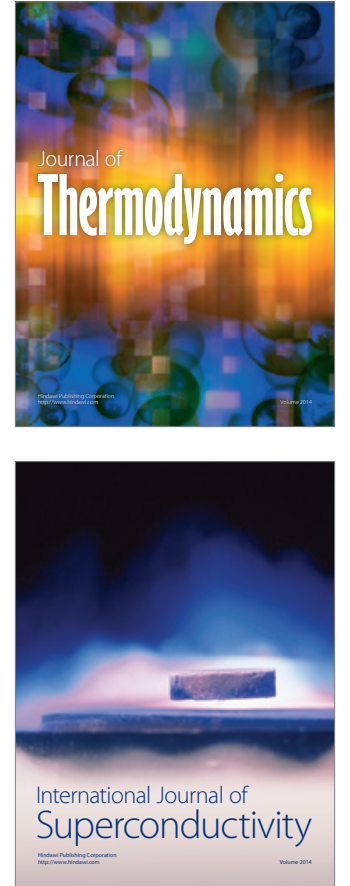
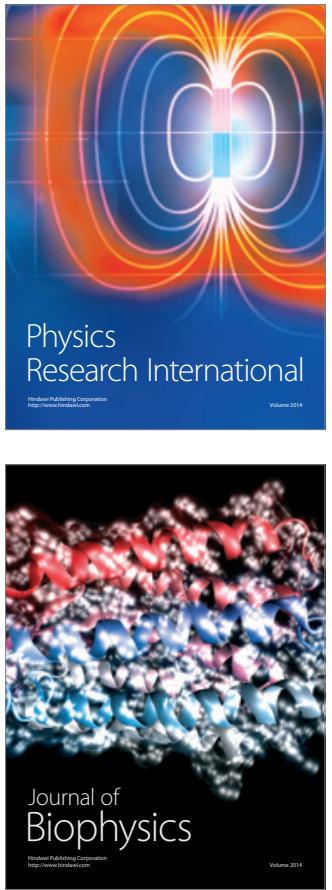
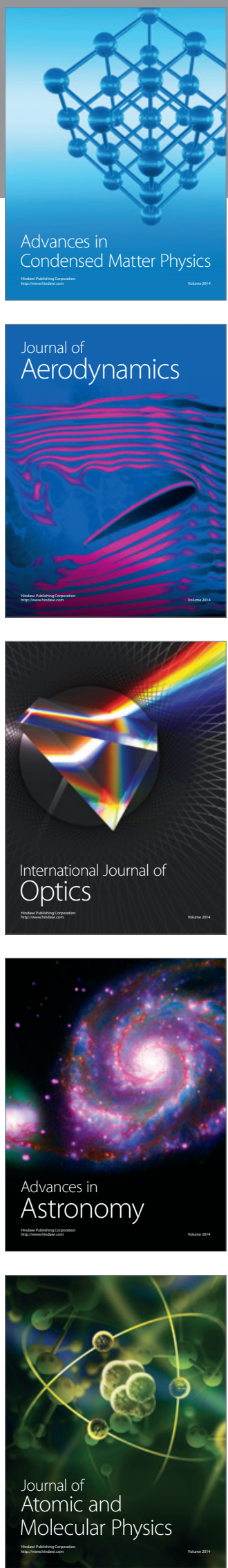